\title{
Aeneas Sylvius Piccolomini et les hommes de lettres de son temps : entre histoire littéraire et théorie littéraire
}

\section{Anne Raffarin}

\section{(2) OpenEdition}

\section{Journals}

Édition électronique

URL : http://journals.openedition.org/cei/83

DOI : $10.4000 /$ cei.83

ISSN : 2260-779X

Éditeur

UGA Éditions/Université Grenoble Alpes

\section{Édition imprimée}

Date de publication : 15 octobre 2011

Pagination : 141-159

ISBN : 978-2-84310-207-3

ISSN : 1770-9571

Référence électronique

Anne Raffarin, «Aeneas Sylvius Piccolomini et les hommes de lettres de son temps : entre histoire littéraire et théorie littéraire », Cahiers d'études italiennes [En ligne], 13 | 2011, mis en ligne le 15 avril 2013, consulté le 27 mars 2021. URL : http://journals.openedition.org/cei/83 ; DOI : https://doi.org/ 10.4000/cei.83 


\title{
AENEAS SYLVIUS PICCOLOMINI ET LES HOMMES DE LETTRES DE SON TEMPS : ENTRE HISTOIRE LITTÉRAIRE ET THÉORIE LITTÉRAIRE
}

\author{
Anne Raffarin \\ Université de Paris 4
}

«Celui-là, dit Aeneas Sylvius, est, d'après mon opinion, véritablement secrétaire apostolique et digne de ce nom important, qui sait choisir les mots et les lier habilement, qui connait l'art de provoquer les passions et de les adoucir, qui fait vibrer dans ses écrits de la finesse, du trait et une culture digne d'un homme libre, qui connaît intimement l'Antiquité et la foule des exemples historiques [...] enfin, qui est capable d'écrire d'une manière habile et ornée tout ce qui concerne l'expédition des lettres. " Cette déclaration, à la fois définition et programme, concentre en elle les maîtres mots de l'humanisme. Les thèmes essentiels de l'éducation des beaux esprits du Quattrocento s'y retrouvent et impliquent des choix de lectures ou de références que les lettres et les Commentarii de Piccolomini permettent de préciser.

Si, à la demande d'Alphonse d'Aragon, Flavio Biondo ${ }^{1}$ s'était fixé pour objectif, entre I447 et I453, d'établir dans l'Italie illustrée une sorte d'annuaire

I. Né à Forlì en 1396, Biondo s'est inséré, dès le début de sa carrière professionnelle, dans les milieux humanistes du Centre-Nord de l'Italie. En I420, il est présenté à Guarino de Vérone immédiatement conquis par les qualités du jeune homme. Le II mars I433, Biondo entre au service de la Curie romaine comme notaire de la Chambre apostolique; il deviendra secrétaire apostolique en I434 puis scriptor des lettres apostoliques en I436. Eugène IV, contraint à l'exil, s'installe à Florence avec les humanistes qui l'accompagnent. Ces derniers côtoient Léon Battista Alberti, Leonardo Bruni, Poggio Bracciolini ou encore Ambrogio Traversari. En I435 à Florence, Biondo rédige son premier ouvrage, le De uerbis Romanae locutionis. Il se lance également dans la grande entreprise des Décades, qui seront publiées entre I437 et I453. Dès le retour d'Eugène IV à Rome en I443, Biondo se consacre à la Roma instaurata (Rome restaurée) de I444 à I446. L'Italia illustrata est composée entre le printemps de l'année I448 et octobre I453, à l'époque où Biondo se voit éloigné de la Curie sous le pontificat de Nicolas V. C'est à la demande du roi de Naples, Alphonse d'Aragon, qu'il rédige une description de l'Italie partagée en dix-huit régions en établissant une sorte d'annuaire des célébrités, des curiosités artistiques et des pôles intellectuels de la péninsule. Il écrit aussi deux ouvrages moins ambitieux : l'Oratio coram serenissimo imperatore Federico en 1452 (discours pour la Croisade) et le De expeditione in Turchos en I453, dédié à Alphonse 
des célébrités de la péninsule de l'Antiquité à la fin du Moyen Âge, tant dans le domaine littéraire, qu'artistique, politique, ou militaire, Piccolomini pour sa part, dans le De uiris illustribus mais aussi dans les Commentarii, semble avoir à cœur de ne retenir que les grandes figures littéraires contemporaines. Plusieurs textes de Piccolomini pourraient s'intituler Italia illustrata, Bohemia illustrata ou Europa illustrata mais c'est le De Europa ${ }^{2}$ qui offre les plus nombreux développements consacrés aux hommes de lettres originaires des régions traversées par l'humaniste.

Pourtant, on ne peut réduire l'ambition de l'humaniste au projet d'accomplir pour l'époque contemporaine ce que Biondo avait accompli dans le cadre plus général de la présentation de l'Italie : dans sa correspondance avec les plus grands humanistes, Piccolomini fait constamment référence aux auteurs de l'Antiquité. Le primat pédagogique détenu par l'Antiquité classique est ainsi assumé et réaffirmé après les traités sur l'éducation déjà nombreux depuis le début du Quattrocento. Au-delà du traditionnel développement sur l'utilité des studia humanitatis qui a été présenté bien avant lui et qui le sera bien après, Piccolomini qui ne se soustrait pas à ce passage obligé, développe une conception des études à la fois traditionnelle, complexe et dynamique : en conseillant à la nouvelle étoile montante de l'Europe qu'incarne Sigismond d'Autriche de s'entourer d'hommes de lettres susceptibles d'illustrer son règne, il annonce entre autres la lettre dans laquelle Flavio Biondo rappelle à Alphonse d'Aragon ${ }^{3}$ que tous les grands hommes se sont entourés d'historiens et de poètes susceptibles de célébrer leur gloire et d'illustrer leurs exploits, faisant implicitement référence au passage du Pro Archia dans lequel Cicéron vante la multiplicité des avantages que présente l'étude des lettres. Mais ce discours se double d'une défense des études littéraires et de la poésie qui n'est pas dénuée d'arrière pensée politique ou, plus généralement, civique, puisque pour les représentants du courant humaniste actif, engagé, le lettré n'est pas un homme de cabinet, voué à vivre dans la seule compagnie des livres. Enfin, l'exhortation à l'étude s'accompagne de recommandations de lectures choisies sans aveuglement admiratif pour les auteurs antiques. Il est recommandé de lire certains auteurs : dans certaines traductions récentes plutôt que dans les traductions approximatives du Moyen Âge pour les auteurs grecs. Nous

d'Aragon. L'année I459 voit la parution d'une autre ouvre majeure dédiée au pape Pie II : la Roma Triumphans (Rome triomphante) dans laquelle il reconstitue la vie et les institutions antiques.

2. Enee Silvii Piccolominei postea Pii PP. II De Europa, edidit Adrianus Van Heck, Città del Vaticano, Biblioteca Apostolica Vaticana, 20oI.

3. B. Nogara, Scritti inediti e rari di Flavio Biondo, Città del Vaticano, Biblioteca Apostolica Vaticana, 1927, p. I47-I53. 
devrons donc nous demander si au-delà des éléments constitutifs d'une histoire littéraire, nous ne trouvons pas déjà en germe dans les textes de Piccolomini, ce qui pourrait relever des prémices de la critique littéraire.

\section{L'actualité littéraire et artistique}

Dans le De uiris Illustribus, Piccolomini procède à une sorte d'imbrication des vies d'hommes de lettres dans les vies des Grands Hommes de l'époque : dans la vie de Nicolas d'Este ${ }^{4}$ est mentionnée l'avancée culturelle qu'a représentée l'arrivée de Guarino de Vérone et de Giovanni Aurispa à Ferrare. Dans la vie de Francesco Foscari ${ }^{5}$, un développement est consacré à Francesco Barbaro et la situation culturelle dans la Venise de l'époque et enfin la biographie de Côme de Médicis ${ }^{6}$ fournit le prétexte à un développement sur Francesco Filelfo, comme si les vies des écrivains et penseurs ne méritaient pas qu'on leur consacre une biographie à part entière. Piccolomini trouve un biais pour aborder indirectement, via la biographie d'un grand prince de l'époque, les qualités de l'œuvre de tel ou tel humaniste. Le seul homme de lettres qui se voit consacrer une biographie dans le De uiris illustribus est Leonardo Bruni ${ }^{7}$, mais elle est déguisée sous celle de l'homme d'état, chancelier de la République de Florence.

Si dans les Commentarii et le De uiris illustribus, il se consacre à l'époque contemporaine, dans la correspondance en revanche, Piccolomini opère un retour vers les auteurs anciens. Dans sa lettre à Wilhlem von Stein, il condamne très sévèrement l'ignorance des princes germains qui consacrent toute leur énergie à des bagatelles et ignorent presque tout des belles lettres en général et des lettres anciennes en particulier. Il dresse de ces princes ignorants un portrait par contraste avec les princes d'Italie qui " ne rougissent pas d'entendre et de connaître des poèmes ${ }^{8}$ ". La littérature antique n'est pas reléguée dans un passé inaccessible et révolu puisque Piccolomini franchit allègrement le fossé d'une dizaine de siècles qui sépare les auteurs antiques de ceux du premier humanisme pour établir des liens de complémentarité: il conseille entre autres à Sigismond de lire la

4. Enee Silvii Piccolominei postea Pii PP. II De viris illustribus, edidit Adrianus Van Heck, Città del Vaticano, Biblioteca Apostolica Vaticana, p. 22.

5. Ibid. p. 29-30.

6. Ibid. p. 33 .

7. Ibid. p. 34-37.

8. "Non erubescunt audire et nosse pæeticam. " Aeneae Sylvii Piccolominei Senensis, Opera omnia, Basileae, per Henrichum Petri, I55I, p. 620. - Toutes nos citations sont empruntées à l'édition de Bâle de I55I. 
Politique d'Aristote dans la traduction de Leonardo Bruni pour apprendre à gouverner la République, l'Économique et l'Éthique pour bien gérer ses affaires familiales, Francesco Barbaro (De re uxoria) pour vivre en bonne intelligence avec sa femme, Plutarque (De liberis educandis) pour éduquer ses enfants, Cicéron (De Amicitia, De Senectute) pour savoir comment se comporter avec ses amis et dans la vieillesse. L'enseignement qu'il faut en tirer, c'est que non seulement l'on peut puiser un savoir encyclopédique dans l'Antiquité mais que comme héritiers de ces prestigieux anciens, les humanistes sont à mettre sur le même plan.

Le De Europa et la correspondance présentent de nombreuses occurrences de l'expression studia humanitatis qui éclairent le rôle central que revêt cette notion dans l'univers intellectuel de Piccolomini ${ }^{~}$. Cette référence à l' humanitas cicéronienne que nous trouvons dans le De oratore n'est pas anodine car elle renvoie à l'idéal de l'homme complet, cultivé, « l'honnête homme " dira le XviI ${ }^{\mathrm{e}}$ siècle, doté de qualités intellectuelles et morales, capable de vivre en société. La définition même des studia humanitatis ne doit pas être restreinte à l'étude des lettres, conception des humanistes de la première génération, comme le suggéraient les écrits de Pétrarque. Si l'on se tourne vers un texte d'Alberti assez peu lu et commenté, on rencontre une acception beaucoup plus large de ce que l'on a coutume aujourd'hui d'appeler « les lettres ${ }^{\text {10 }}$ ». Si Alberti ne fait pas coïncider litterae et studia humanitatis, c'est pour englober dans les litterae des sciences telles que le droit ou la médecine. On pourrait avancer, pour synthétiser cette définition des litterae, qu'Alberti les entend au sens de sciences libérales et d'arts libéraux ${ }^{\text {II. }}$. Piccolomini est l'héritier de cette synthèse et de cette conception englobante, mais ce qui le préoccupe surtout, dans le vieux débat stoïcien de la participation du sage à la vie de la cité, c'est que l'honnête homme formé aux studia humanitatis et aux arts libéraux doit s'engager, à moins qu'il ne renverse la perspective en disant que celui qui est amené à s'engager doit être un honnête homme.

9. Entre autres : Opera omnia, ouvr. cité, p. 454 et p. 459; De Europa, ouvr. cité, p. 56 ; p. 203 ...

Io. J. M. Mandosio, "La classification des sciences et des arts chez Alberti ", dans Leon Battista Alberti (Actes du congrès international de Paris, IO-I5 avril 1995), sous la direction de F. Furlan, P. Laurens, S. Matton, Nino Aragno, Turin / Paris, Vrin, 2000, p. 644, cité par C. Carraud, dans Leon Battista Alberti, Avantages et inconvénients des lettres, trad. de C. Carraud et R. Lenoir, Grenoble, J. Million, 2004, p. I7I : "Par le terme générique de "lettres", Alberti ne désigne pas seulement les études littéraires, mais également la pratique des arts et la connaissance des disciplines (V, 3I). "

II. L. B. Alberti, Avantages et inconvénients..., ouvr. cité, V, 3I; 36; 38. 


\section{L'actualité philologique}

La transmission de ces textes issus de l'Antiquité n'est pas un aspect de l'histoire littéraire que néglige Piccolomini. Il s'inscrit même dans la lignée des humanistes qui suivent de près l'activité philologique. Une lettre de Giovanni Campisio dans le courant de l'année I444, fait état des difficultés qu'il a éprouvées à trouver un exemplaire de la Politique d'Aristote à vendre à Rome, alors qu'il en avait repéré un à Sienne. Il précise qu'à Florence, on trouve quantité de livres à acheter et il souligne le succès des textes grecs que Leonardo Bruni a traduits. Il a donc décidé d'en faire copier un exemplaire pour son correspondant qui ne pouvait plus patienter, répondant ainsi à une demande qu'avait formulée Piccolomini dans une lettre datée de la fin de l'année i443. C'est auprès du Cardinal Colonna que Campisio trouva l'exemplaire recherché et le fit copier :

Erit autem hic liber in papyro propterea quod scriptorem nondum habere potui qui in pergameno ipsum scriberet ea litera et eo tempore quo conueniret et oporteret ${ }^{12}$.

Nous apprenons également, par une lettre de juillet $\mathrm{I} 443^{13}$ que Piccolomini avait demandé à Antonio Loschi da Vicenza de l'aider à recopier les discours de Cicéron dont il avait lui-même entrepris la retranscription pendant son séjour à Bâle. Lorsqu'il a cherché à se faire remplacer dans cette tâche fastidieuse, il n'a trouvé aucun moine qui lui parût suffisamment lettré pour recopier sans faute, ce qui en dit long sur les préjugés des humanistes envers les moines, grossiers héritiers des âges obscurs, inaptes à retranscrire la belle langue. Le même type de reproches se rencontre dans les lettres du Pogge.

\section{L'histoire littéraire}

Cet intérêt pour la transmission et la traduction induit et justifie des choix en matière d'histoire littéraire. Constituant en quelque sorte un prélude littéraire à ce qu'écrira Vasari dans les Vies au Xvi siècle, Piccolomini établit les fondements de l'histoire littéraire sur l'idée de progrès successifs accomplis par des auteurs de plus en plus géniaux. On sait que c'est Boccace qui, en appliquant la théorie de l'histoire de Pétrarque à ce que Dante dit de Giotto, remit en l'honneur la notion selon laquelle la peinture s'est développée parallèlement à la littérature, élargissant ainsi le concept du

I2. Opera omnia, ouvr. cité, p. 745 .

I3. Ibid., p. 600. 
grand renouveau en le faisant passer de l'expérience littéraire à l'expérience visuelle. De même que Giotto a dépassé Cimabue, de même Coluccio Salutati incarne-t-il la figure du passé par rapport à un Bruni ${ }^{14}$. Un siècle plus tard, Piccolomini reprend à son compte le parallèle et la théorie de l'évolution qu'il induit. D'ailleurs dans une lettre de I444 adressée à Nicolas d'Ulm, il formule sur la corrélation entre les arts une opinion qui sera reprise par Alberti et à laquelle Lorenzo Valla donnera un nouveau souffle dans les Elegantiae. Il écrit en effet :

Ces deux arts ont en effet des affinités. Le génie de la peinture tout comme celui de l'éloquence exigent, à l'opposé du vulgaire, de la noblesse et du sublime. Il est extraordinaire de remarquer qu'alors que l'éloquence était florissante, la peinture l'était également, comme le prouvent l'époque de Démosthène et de Cicéron. Quand l'éloquence s'est effondrée, la peinture a sombré. Lorsqu'elle s'est réveillée, la peinture aussi a émergé. Nous constatons que durant deux cents ans la peinture a été exécutée sans aucun art. Or, les écrits de cette époque sont bruts, ineptes, sans élégance. Après Pétrarque, les lettres ont émergé. Après Giotto, la main des peintres s'est affermie; nous les voyons aujourd'hui l'une et l'autre parvenue à un niveau artistique très élevé ${ }^{15}$.

Piccolomini s'essaie également à une reconstruction de l'histoire littéraire présentée parallèlement à l'évolution des arts, dans la lettre qu'il adresse à Grégoire de Hambourg lorsqu'il explique que l'Italie a connu une époque où :

Ayant enterré l'éloquence, elle obéissait à la justice des barbares. Dans les cent ans qui précèdent et même auparavant dans les trois cents ou quatre cents ans qui ont précédé, on ne trouverait pas un orateur qui ait prononcé un discours élégant et clair. Il est arrivé la même chose à la peinture et à la sculpture. Si tu observes des peintures ou des sculptures d'il y a deux cents ou trois cents ans, tu ne trouveras pas des visages d'hommes mais de monstres ou de créatures fabuleuses [...] Mais voici qu'ont refleuri l'art de la sculpture et celui de la peinture. Avec eux s'est réveillée l'éloquence et elle brille aujourd'hui de tout son éclat parmi les Italiens. J'espère qu'il en sera de même chez les peuples teutons ${ }^{16} \ldots$

I4. Voir M. Miglio, Biografie e raccolte biografiche nel Quattrocento italiano, in Atti dell'Accademia delle Scienze dell'Istituto di Bologna. Classe di Scienze morali, LXIX, Rendiconti, 63, 1974-1975, fasc. I, p. I66-199.

15. Opera omnia, ouvr. cité, p. 646 : "Amant enim se artes hae adinuicem. Ingenium pictura expetit, ingenium eloquentia cupit non unlgare sed altum et summum : mirabile dictu est, dum vigit eloquentia, uigit pictura, sicut Demosthenis et Ciceronis tempora docent. Postquam cecidit facundia, iacuit et pictura. Cum illa reuixit, haec quoque caput extulit. Videmus picturas ducentorum annorum nulla prorsus arte politas. Scriptia illius aetatis rudia sunt, inepta, incompta. Post Petrarcham emerserunt litterae. Post Iotum surrexere pictorum manus, utramque ad summam iam nidemus artem peruenisse."

16. Ibid., p. 647 : «Mirabar cur hodie nibil eloquentiae his in regionibus eluceret. Sed uenit in mentem apud Italos quoque tempus fuisse quo sepulta dicendi facultate barbarorum iustitia dominabatur. Ante centum ferme annos et antea trecentis quadringentisque non inuenies qui per Italiam sermonem habuerit tersum et lucidum. Sic pingendi sculpendique accidit arti. Si ducentorum trecentorumque annorum aut sculpturas intueberis aut scripturas inuenies non hominum sed monstrorum portentorumque facies. [...] Sed ecce iam reuixerunt sculpendi pingendique 
Les peintres avaient fait leur entrée dans le panthéon des hommes illustres dès le De uiris illustribus de Bartolomeo Facio (I456), recueil entièrement consacré aux contemporains. À partir de cette date, l'idée que l'âge moderne dépasse l'âge obscur qui a précédé, auparavant implicite, devient explicite et donne lieu à une sorte de querelle des Anciens et des Modernes dont un autre témoignage contemporain est le De uiris illustribus de Piccolomini. Mais finalement du point de vue de l'histoire littéraire, le De Europa fournit bien plus d'éléments sur les hommes qui ont fait l'histoire littéraire du Quattrocento.

\section{De l'histoire littéraire à la critique littéraire ou la formulation de jugements critiques.}

L'histoire littéraire s'inscrit dans le cadre plus général de la vision de l'histoire propre à Piccolomini : de même que ce sont les grands hommes qui font l'histoire, ce sont quelques grandes figures qu'il convient d'identifier et de mettre en exergue pour construire l'histoire littéraire. Mais ce qui distingue Piccolomini de ses prédécesseurs et fait l'intérêt de certains passages de ses Commentarii mis en relation avec sa correspondance, c'est la formulation de jugements personnels sur le style des auteurs. Jamais Biondo qui ouvrit la voie à ce que l'on pourrait qualifier avec prudence l'histoire littéraire, n'exprime d'appréciation sur le talent de tel ou tel auteur. Tous ceux qu'il choisit de citer sont indistinctement remarquables, brillants ou exceptionnels. Quant aux chroniques d'auteurs comme Giovanni Villani, elles signalent plutôt les grands auteurs qui ont assuré le rayonnement de leur cité et qui incarnent une sorte de génie national. Giannozzo Manetti ${ }^{17}$ qui fut le premier à regrouper les vies des Trois Couronnes de Florence dans les Vies de Dante, Pétrarque et Boccace ${ }^{18}$ choisit de rapporter le plus d'anecdotes possible ${ }^{19}$ en utilisant des témoignages qui, au Trecento, avaient contribué à créer une aura légendaire autour de Dante. Le patriotisme de Manetti culmine avec la célébration de Pétrarque et de Boccace comme les hérauts

disciplinae. Reuixit etiam eloquentia et nostro quidem seculo apud Italos maxime floret. Spero idem in Theutonia futurum...»

17. Giannozzo Manetti (Florence 1396-Naples I459) étudia le latin, le grec et l'hébreu avec son ami Vespasiano da Bisticci avant d'entrer dans la carrière politique et diplomatique. En I440, il rédige une Vita Socratis et Senecae et la même année, les Vies de Dante, Pétrarque et Boccace.

I8. Biographical writings, S. U. Baldassari et R. Bagemihl (éd. et trad.), "The I Tatti Renaissance Library ", Harvard/Cambridge, 2003, p. 8-IO5.

19. Ibid., introduction p. XII-XIII. 
d'une Renaissance durable de la culture ayant son origine à Florence ${ }^{20}$. Dans le registre du style, si Manetti ${ }^{21}$ affirme l'incontestable supériorité des trois auteurs sur tous les autres écrivains en langue vulgaire, dans le domaine de la littérature latine, en revanche, il souligne leur faiblesse et justifie le peu de considération dont ils font l'objet auprès des hommes cultivés. Aucun jugement littéraire sur leur style, ni sur la capacité de chacun d'entre eux à plaire ou émouvoir.

Dans les Commentarii de Piccolomini, certaines villes, comme Florence, suscitent la matière de développements fournis sur l'histoire littéraire. Au livre 30 du chapitre II, il fait écho aux discussions qui ont traversé une bonne partie du siècle sur les Trois Couronnes de Florence ${ }^{22}$ :

Dans le passé il y eut à Florence de nombreux hommes illustres dont le nom est encore vivant aujourd'hui; mais Dante Alighieri semble tous les surpasser, avec son insigne poème et sa fameuse création du Paradis, de l'Enfer et du Purgatoire d'où émane le parfum d'une sagesse presque divine, même si comme tout homme, il commit des erreurs. Juste après lui vient Pétrarque, auquel il serait difficile de trouver un égal si ses œuvres en latin pouvaient être comparées à celles écrites en toscan. À la troisième place, nous mettrions sans faute Boccace, bien qu'il fût un peu trop licencieux et d'un style quelque peu relâché. "Il est suivi par Coluccio qui écrivit aussi bien en prose qu'en vers, mais dans une poésie adaptée à son époque et rude pour la nôtre. Il fut à la tête de la chancellerie de Florence et Giangaleazzo, duc de Milan, avait l'habitude de dire que la plume de Coluccio lui était plus nuisible que trente cohortes de cavaliers florentins. C'était en fait un homme sage et bien qu'il n'eût pas un style élégant, il était tout à fait rompu aux tours rhétoriques qui savent habituellement émouvoir les hommes et, par son écriture, il les touchait avec discrétion ${ }^{23}$.

Entre les courtes biographies de De uiris et le développement des Commentarii, une évolution s'est incontestablement produite puisque dans le premier texte, le développement sur Florence et Bruni se terminait de façon lapidaire : Bruni s'est élevé au niveau de Cicéron alors que dans le second texte, le style des Trois Couronnes de Florence est abordé avec beaucoup plus de précision et de nuance. Que s'est-il produit dans l'intervalle d'une douzaine d'années qui sépare les deux textes? Les controverses humanistes sur la langue et la référence permanente aux auteurs florentins fondateurs de la nouvelle littérature en langue vernaculaire et du dolce stil

20. Ibid., introduction, p. XV.

2I. Ibid., p. $4 \$_{3}$.

22. Pius II, Commentaries, M. Meserve et M. Simonetta (éd.), Cambridge, MA, Harvard University Press, 2004, "The I Tatti Renaissance Library ", p. 322.

23. Mémoires d'un pape de la Renaissance. Les Commentarii de Pie II, présentés et annotés par I. Cloulas et Vito Castiglione Minischetti, traduction (partielle) des Commentarii de Pie II, Paris, Tallandier, 2oor, Livre II (I458-I459), p. I44-I45. 
nuovo ont donné lieu à des échanges de lettres ou de traités qui n’ont pas pu échapper à la vigilance d'un Piccolomini. À mi-chemin entre les deux textes, dans la lettre à Sigismond, il prend position sur l'histoire littéraire en faisant de Bruni et de Chrysoloras les grands acteurs de la renaissance des lettres; il y développe et y dépasse à la fois le passage du De uiris dans lequel il range Coluccio Salutati dans l'âge des ineptias et du squalor. Or, si l'on se rappelle ce qu'écrivait Bruni dans son Commentarius rerum suo tempore gestarum dans les premières années du siècle, on trouve formulé le jugement que Piccolomini reprend à son compte : "Postquam Franciscus Petrarcha, omisso temporis sui squalore, priscam coepit eloquentiam imitari ${ }^{24}$. "

C'est véritablement le duo complémentaire Chrysoloras-Bruni qui revêt, sous la plume de Piccolomini, le rôle de promoteurs et de rénovateurs de la belle langue et des arts libéraux. Piccolomini faisait partie de la génération qui a eu la chance d'évaluer les progrès que l'apprentissage du grec a fait faire à la culture dès les débuts de l'humanisme. Dans sa correspondance, les mentions des lettrés cultivés qui connaissaient le grec sont nombreuses et destinées à distinguer des savants. Cette célébration du rôle joué par Chrysoloras lui avait en quelque sorte été soufflée par Bruni dans le Commentarius rerum suo tempore gestarum ${ }^{25}$ et bien sûr dans la Laudatio Florentinae Vrbis. Bruni avait décrit le petit cercle de traducteurs qui s'était constitué autour du savant byzantin (Giovanni Aurispa, Guarino de Vérone, Palla Strozzi, Pier Paolo Vergerio) qui suscitent tous l'admiration d'un Piccolomini et sont mentionnés dans ses lettres, indirectement ou comme destinataires.

La réponse à la lettre de Leodrisio Crivelli qui lui offrit en 1457 sa traduction d'une lettre de saint Chrysostome à l'évêque Cyriaque, patriarche de Constantinople, illustre bien l'admiration qu'avait Piccolomini pour les hellénistes confirmés :

J'ignorais que tu savais le grec : j'ai découvert ta traduction avant d'apprendre que tu connaissais cette langue. Je t'estime en proportion de ce que représente le fait de savoir deux langues plutôt qu'une. Je n'ai pas d'avis sur le texte grec, mais ce qui a été traduit du grec au latin, si je comprends bien, est tout à fait admirable, élégant, suave et brillant. Je me réjouis que tu sois parvenu à une telle maîtrise de cette discipline que tous doivent s'accorder à louer tes écrits ${ }^{26}$.

24. Opera omnia, ouvr. cité, p. 600 (Lettre à Sigismond d'Autriche, décembre I443).

25. Rerum italicarum scriptores, L. A. Muratori (éd.), vol. I9, Milan, Società Palatina, I73I, p. 920.

26. Opera omnia, ouvr. cité, p. 804 : «Nesciebamus te ante Graeci sermonis gnarum esse : prius translationem hanc uidimus quam te graece doctum audiremus. Tanto te magis existimamus quanto magis est duas linguas quam unam nosse. Non est nobis iudicium de Graecis. At quod latine scriptum est ex Graeco uersum, si quid est quod sentimus, admodum laudabile est, tersum, suaue, nitidum. Gaudemus te huic doctrinae profectum ut laudari ab omnibus tua scripta debeant. " (Lettre d'octobre I457.) 
Reste à examiner le jugement de l'auteur lui-même sur son œuvre. Tout d'abord, nous rencontrons un jugement sur ses travaux historiques, associé au verdict pour le moins sévère qu'il porte sur les œuvres de Flavio Biondo :

Il écrivit une histoire universelle depuis les empereurs Honorius et Arcadius, c'est-àdire depuis le commencement du déclin de l'empire romain jusqu'à son époque : œuvre certes sérieuse et utile mais qui aurait besoin d'être polie et corrigée. Peut-être dira-t-on la même chose de notre œuvre, et non sans raison parce que, même si les faits que nous narrons sont véritables, on les trouvera tantôt dignes d'attention, tantôt sans intérêt, et que, l'élégance du style nous faisant défaut, on estimera que nous avons écrit une histoire indigeste et grossière. Peut-être un autre mettra-t-il en lumière ce que Biondo et nous-même avons composé et recueillera-t-il les fruits du labeur d'autrui ${ }^{27}$.

Mais le passage le plus étonnant est extrait de sa correspondance. Dans la lettre au cardinal primat de Pologne Zbegniew Olesnicki qui souhaitait obtenir un recueil des lettres de Piccolomini alors cardinal de Sienne, ce dernier formule un jugement sur son propre style qui pourrait presque se résumer par la formule " ce qui se conçoit bien s'énonce clairement "; celleci permet à Piccolomini de justifier sa sécheresse et son manque d'élégance :

Je mesure à quel point la grâce de mon discours est ténue, pauvre et mon bagage lexical dépourvu d'ornement. [...] J'avoue pourtant que comme je m'exprime à nu et à cœur ouvert, je n'emploie pas d'ornement, je rejette tout voile et je ne fais pas d'effort lorsque j'écris puisque je ne vise pas de sujets très élevés ni de sujets inconnus de moi : je ne transmets que ce que j'ai appris. Il se donne facilement à comprendre aux autres, celui qui est en cohérence avec lui-même, en revanche quiconque garde une part d'ombre pour soi-même ne peut apporter des lumières à autrui. J'évite la complexité et les longues périodes. Si des termes élégants se présentent, je ne répugne pas à les disposer, dans le cas contraire, je ne vais pas chercher plus loin, j'ordonne ceux dont je dispose pour que le lecteur comprenne. C'est mon seul objectif. Je me rends bien compte que mon propos est rude et peu élaboré et qu'il n'est pas digne de retentir aux oreilles des savants ${ }^{28}$.

Ce passage empreint de fausse modestie présente un intérêt en ce qu'il constitue une analyse éloquente de l'efficacité du style. Le but de Piccolomini est bien de démontrer et de se faire comprendre pour persuader, comme si le docere venait au premier plan, le placere au second.

27. Mémoires d'un pape..., ouvr. cité, Livre XI (I462-I463), p. 4II. Pour le texte original, voir : Enea Silvio Piccolomini-papa Pio II, I Commentarii, L. Totaro (éd.), Milan, Adelphi, II, p. 2257.

28. Opera omnia, ouvr. cité, p. 934 : "Sentio quam tenuis est mihi uena dicendi, quam breuis et inornata uerborum suppellex: nulli de me magis quam mihi credo: fateor tamen quia nundus sum et aperte loquor non utor phaleris, uestem omnem reiicio, nec laboro cum scribo quoniam non attingo res altiores et mihi non cognitas : trado quae didici. Facile se caeteris intelligendum praebet, qui se ipsum tenet, praebere alteri lucem nequit, qui sibi tenebrosus est. Fugio nodositatem et longas sententiarum periodos. Si assunt elegantia uerba, non refugio illa contexere, si minus non quaero remotius, praesentibus utor, ut intelligat. Id solum mibi studium est. " (Lettre de novembre I463.) 
L'indulgence qu'il invoque pour l'absence d'ornements de son style est justifiée par le souci de clarté et d'efficacité, par la préoccupation constante de nommer et de désigner précisément des notions. Mais à un deuxième niveau, on peut trouver un intérêt d'un autre ordre à cette analyse : par sa place et sa forme qui est presque celle de la confession, elle définit l'espace de la lettre comme lieu de l'analyse littéraire, instaurant une sorte d'espace littéraire nouveau. L'espace de la lettre peut donc être défini comme un espace littéraire privilégié dans lequel se déploient des expériences que l'auteur n'aurait pas tentées dans le cadre plus rigide d'un ouvrage en prose ou en vers, dédié à un grand personnage. La lettre constitue un espace privilégié d'expression sur la littérature. C'est dans une lettre adressée à Piccolomini par son neveu (Gregorio Lolli Piccolomini, par ailleurs correspondant de Biondo) vraisemblablement en I443 ou I444, que se trouve un point de vue sur les fonctions de l'art épistolaire qui mutadis mutandis, rappelle ce que Cicéron écrit sur l'amitié ${ }^{29}$ ou Alberti sur la peinture ${ }^{30}$ :

Il est une ancienne formule très juste de saint Jérôme : entre autre utilité, les lettres ont ce mérite qu'elles rendent présents les absents. C'est même admirable : nous nous adressons aux absents, nous voyons ceux qui sont de l'autre côté des mers, les pensées silencieuses de notre esprit sont dessinées par des courbes et des lignes, qui surpassent en antiquité et en utilité pour le genre humain toute autre invention ${ }^{31}$.

La préface au commentaire du livre d'Antonio Panormita sur Alphonse d'Aragon est également rédigée comme une lettre ou plutôt comme un feuilleton épistolaire, avec des séquences. Le jugement formulé sur les qualités de Panormita est d'autant plus intéressant que pressentant intuitivement

29. De amicitia, XXIII : "Verum enim amicum qui intuetur, tamquam exemplar aliquod intuetur sui. Quocirca et absentes adsunt et egentes abundant et imbecilli valent et, quod difficilius dictu est, mortui vivunt ; tantus eos honos, memoria, desiderium prosequitur amicorum. " ( En effet, observer un véritable ami équivaut à observer quelque version exemplaire de soi-même : les absents sont alors présents, les indigents sont riches, les faibles pleins de force et, ce qui est plus difficile à expliquer, les morts sont vivants : tant le respect, le souvenir, le regret de leurs amis continue de leur être attaché. ")

30. De pictura, II, $25:$ : [...] hoc loco ostendendum censeo quam sit pictura non indigna in qua omnem operam et studium consumamus. Nam habet ea quidem in se uim admodum diuinam non modo ut quod de amicitia dicunt, absentes pictura praesentes esse faciat, uerum etiam defunctos longa post saecula niuentibus exhibeat, ut summa cum artificis admiratione ac uisentium uoluptate cognoscantur. " (" [...] Il faut montrer ici combien la peinture n'est point indigne que nous lui consacrions tout notre travail et notre application. C'est qu'elle a en elle une force toute divine qui non seulement comme on le dit de l'amitié, permet à la peinture de rendre présents les absents, mais encore de faire surgir après de longs siècles les morts aux yeux des vivants : si bien qu'on les reconnaît, ce qui vaut au peintre la plus grande admiration et suscite le plus grand plaisir chez ceux qui regardent. ")

3I. Opera omnia, ouvr. cité, p. 750, epistola CLXXIX : "Hieronymi uetus admodum sententia est probatissima, litterarum inter caeteras hanc esse utilitatem, ut absentes praesentes faciant. Praeclare quidem: Nam ad absentes his loquimur, uidemus transmarinos, animique nostri cogitatio sine uoce, notarum picturis atque lineamentis denuntiatur, quo certe utilius quicquam aut humano generi antiquius non facile inuestigari posset. " 
le lien entre le fond et la forme, Piccolomini lie le style du panégyrique au style de cet éminent personnage qu'est le roi de Naples :

Tu m'as demandé de relire et de corriger ce que tu avais écrit au sujet de cet immense et excellent souverain. Lire, je le pouvais, et je l'ai fait, mais corriger, je ne le pouvais pas. En effet, quel texte écrit de ta main mérite-il correction? Comme on dit couramment, il n'est rien qui puisse être mieux formulé. [...] Malheureusement, à mon grand regret, j'ignore le grec, mais si mon jugement sur ce qui est écrit en latin est de quelque valeur, ni Xénophon traduit en latin n'arrive à la cheville d'Antonio, ni Socrate ne peut rivaliser avec Alphonse. Philelpho a traduit en latin les apophtegmes de Plutarque. On y retrouve résumés les paroles et les exploits dignes de mémoire des grands hommes que non seulement l'Orient, mais aussi la Grèce et Rome ont produits. Mais ton livre expose des exploits plus grands et plus illustres encore du seul Alphonse. Heureux homme à qui est échu le privilège de décrire un si grand prince, heureux Alphonse qui a trouvé en toi un écrivain si talentueux pour célébrer ses hauts faits. Tu ne pouvais trouver matière plus noble ni lui plus élégant auteur ${ }^{32}$.

\section{Le portrait de l'homme de lettres en action. Le nouvel idéal du lettré engagé}

Ce face à face avec les textes antiques fait éclore l'idée que les studia humanitatis servent de fondement à l'action de l'homme dans le monde et impliquent un choix et une prise de position dans les débats du moment sur les rapports et la compatibilité entre vie active et vie contemplative. Piccolomini, dans ce registre, est bien plus proche de l'incitation à l'action formulée par Alberti dans le De commodis que des définitions de l'otium litteratum, du retrait et de la vie solitaire énoncées et reprises de Cicéron à Pétrarque. L'action est un impératif qui contraint l'homme de lettres à devenir un homme engagé dans la vie politique et sociale. Cette idée déjà présente à la fin du premier livre du De Officiis où Cicéron fait s'articuler les officia des lettrés avec ceux de la communauté des hommes n'a rien perdu de son actualité.

32. Ibid., p. 472 : "Nam quae de rege maximo et optimo olim scripseras, ut legerem corrigeremque iussisti. Legere potui, quod feci, corrigere uero non potui. Nam quid est quod manu tua emissum correctione indigeat? Vulgo dicitur, nibil dictum esse quod nequeat dici melius. [...] Ignoramus etiam damno notro graecas literas. De latinis si quod est iudicium nostrum, neque ille translatus se Antonio, neque Socratem Alphonso parem facit. Nam et tu Xenophontem hunc eloquentia superas et Alphonsus sapientia Socratem antecedit. Quid plura? Apophtegma Plutarchi Philelphus in Latinum uertit. Dicta illic factaque memoratu digna breuiter comprehensa sunt illustrium uirorum, quos non oriens modo, sed Graecia simul et Roma protulit. Maiora tamen et illustriora de uno Alphonso tuus liber explicat. Felix tu igitur cui tantus princeps scribendus occurrit, felix Alphonsus qui te ad consecranda eius gesta idoneum offendit. Nam neque tibi materiam uberiorem inuenire neque illi scriptorem elegantiorem obtinere facile fuerat. " 
Il faut également se souvenir que ces discussions liées à l'engagement de l'homme de lettres dans la vie de la cité avaient déjà nourri un texte peu connu, récemment ${ }^{33}$ traduit et peu commenté, Les entretiens de Camaldoli de Cristoforo Landino (I475), entretiens auxquels participait précisément Leon Battista Alberti. Il n'est pas illégitime d'établir un parallélisme entre Frédéric de Montefeltre qui prit en main les destinés de l'Etat d'Urbino à partir de I444 et fut considéré par nombre d'humanistes comme prince modèle, ami des hommes de lettres, et l'idéal que l'humaniste Piccolomini représentait à Sigismond d'Autriche. Dès la dédicace, Landino loue conjointement en Frédéric de Montefeltre l'homme de lettres et l'homme d'action, le grand chef de guerre vainqueur si attaché au travail intellectuel ${ }^{34}$ qu'il avait fait construire dans son château d'Urbino l'une des bibliothèques les plus admirables de la Renaissance, comparable à celle des Médicis à Florence. Laurent de Médicis n'est pas absent de ce texte puisqu’à la dédicace à Frédéric succède un dialogue entre Laurent lui-même et Leon Battista Alberti dont l'arrivée inopinée permet de traiter la question des rapports entre le philosophe et l'homme d'État, la pensée platonicienne étant devenue familière aux florentins de l'époque grâce aux travaux de Marsile Ficin. L'argumentation développée par Alberti, suivie de celle de Laurent, vise à défendre le principe d'un engagement du lettré au service de la communauté des citoyens ${ }^{35}$. Cette problématique se trouve au cour des réflexions que Piccolomini développe dans sa correspondance.

Les pédagogues humanistes ont, dans leur immense majorité, jugé que l'éducation devait revêtir une fonction morale permettant aux jeunes gens de se préparer à exercer des activités civiques au service de leur cité ou de leur pays. Piccolomini s'inscrit dans la lignée des humanistes auteurs de traités pédagogiques, vers I450, avec le De liberorum Educatione, mais pour cerner ses intentions, il faut établir un rapprochement avec le genre littéraire du miroir des Princes, très pratiqué au Moyen Âge mais qui n'était guère en vogue dans la première phase du Quattrocento; dans la seconde moitié du siècle, en revanche, le Prince devint l'un des sujets de discussion

33. Cristoforo Landino, Disputationes camaldulenses, C. Carraud (trad.), Conférence, 200I, $\mathrm{n}^{\circ} \mathrm{I} 2$, p. 624-675.

34. Ibid., p. 630: "Tu avais déjà très tôt une admirable compréhension des poètes, tu possédais toute l'histoire, tu connaissais par cœur les préceptes de l'art de la parole et tu mettais même le plus grand soin à t'y exercer; plus tard, tu consacras toute ton attention à la philosophie, imitant en cela l'exemple des plus grands capitaines, pour avoir lu qu'Alexandre le Grand avait eu pour maitre Aristote, Scipion l'Africain, le stoïcien Panétius... »

35. Ibid. p. 652-653 : "Mais si, parmi tous ces gens, on voyait votre sage perdu dans ses pensées et bâillant d'inaction, replié seul dans sa bibliothèque d'où il ne sort jamais [...] et à l'écart de toute activité publique et même privée, quel rôle lui donner dans l'État, quel symbole apporter par son entremise à la vie humaine? ” 
favori suscitant une grande diversité d'écrits sur ce thème ${ }^{36}$. Si les premiers écrits sur le Prince traitent de son éducation, et c'est dans ce registre que nous nous situons, les écrits de la fin du siècle, en revanche, proposent une réflexion sur le gouvernement princier.

Les réflexions de Piccolomini mettent en lumière sa vision utilitaire de l'étude des belles lettres pour les hommes de pouvoir. La perception que l'on peut avoir de son état d'esprit d'après quelques extraits bien connus de la correspondance est complétée par certains passages de l'Historia de Europa sur Ferrare ou Florence par exemple. À Ferrare, chez le marquis d'Este la discussion qui se développe autour des qualités respectives des philosophes grecs, aboutit à la conclusion suivante : les latins de l'époque qui sont à la fois imprégnés des belles lettres et impliqués dans l'action sont bien supérieurs aux Grecs qui ne savaient que parler. Piccolomini introduit deux exigences supplémentaires. La première est de se pénétrer de la pensée des auteurs que l'on étudie : «Et je ne te déclarerai pas lettré parce que tu parles en latin ", prévient-il dans la lettre à Sigismond. "Même si cela est déjà beau, c'est aussi à la portée des corbeaux et des pies. Non, je te déclarerai lettré lorsque tu comprendras les orateurs, lorsque tu connaîtras les philosophes, lorsque tu comprendras les poètes ${ }^{37}$. " La deuxième exigence consiste à mettre en œuvre le fruit de ses lectures dans l'action politique : Piccolomini recommande à Sigismond de ne pas se couper de la société, de ne pas abandonner l'action au profit de la lecture. Il insiste sur l'idée que la littérature n'est pas une fin en soi mais il en construit une vision pragmatique :

Je ne souhaite pas que tu consacres des jours et des nuits aux livres. Mais je revendique du moins que tu réserves une heure d'un jour qu'il te plaira pour les belles lettres. [...] Car il est érudit celui qui, doté d'un talent naturel, a consacré une attention soutenue aux auteurs qui sont les maîtres de leur art, qui, après avoir parcouru de nombreux volumes en a gardé l'essentiel en mémoire et qui n'est pas seulement imprégné des auteurs sacrés mais également des auteurs profanes. Parmi ceux-ci, je voudrais que tu en choisisses un avec lequel tu puisses dialoguer librement [...]. Pour ma part, j'approuve le fait que ta demeure soit remplie de soldats. Ils protègent la patrie et c'est un honneur pour un prince de gagner la gloire des armes, mais que personne auprès de toi ne soit cultivé, je ne l'approuve ni le recommande. De même que tu entretiens

36. Leonardo Bruni, De studiis et litteris liber (I444) pour Battista Malatesta d'Urbino; Piccolomini, la lettre à Sigismond (I443) et le De liberorum educatione pour Ladislas de Hongrie (I449); Francesco Filelfo, De morali disciplina et lettre à Giovanni Sforza (circa I480); Bartolomeo Platina, Principis Diatyposis (I48I); Francesco Patrizi, De regni et regis institutionel De institutione reipublicae (I494); Giovanni Pontano, De principe liber (circa I503); Nicolas Machiavel, Il Principe (I513).

37. Opera omnia, ouvr. cité, p. 602 : «Nec enim literatum te dicam quod latinum pronunties. Nam etsi pulchrum est, coruis et picis datur... Pulchrius tamen erit et tunc te literatum nocitabo cum oratores intelliges, cum philosophos nosces, cum poetas tuapte percurres. " 
des soldats, tu pourrais entretenir des hommes de lettres qui t'enseigneraient les distinctions et les limites entre le juste et l'injuste et feraient de toi un homme parfait sous tous rapports ${ }^{38}$.

Après avoir évoqué les héros de l'histoire antique grecque et latine, il évoque des exemples d'hommes illustres de son époque qui sont de grands lecteurs, de vrais amateurs des belles lettres mais aussi des hommes d'action, dans une description presque comique tant elle est excessive : Leonello d'Este qui écrit comme Cicéron, Alphonse d'Aragon qui emporte ses livres sur le champ de bataille rappelant la figure tutélaire de Jules César à propos duquel il écrit : "Is enim cum in Gallia bellum gereret, diem armis, noctem litteris dedit ${ }^{39}$. " D'ailleurs, on remarque que dans le commentaire aux livres d'Antonio Panormita, De dictis et factis Alphonsi regis memorabilibus commentarius, Piccolomini ajoute ce commentaire au portrait que brosse Panormita de ce grand imperator savant et philosophe :

Alors que j'accompagnais Alphonse de Baïes à Pouzzoles et qu'il m'entretenait des livres, il déclara avoir lu la cité de Dieu de saint Augustin, traduit en français, et dans la préface duquel il est écrit : "Un roi sans instruction n'est rien d'autre qu'un âne couronné $4^{40}$.»

La permanence des valeurs héroïques au Quattrocento s'exprime dans le portrait du doctus imperator que brosse Piccolomini dans sa lettre à Sigismond ${ }^{41}$. On retrouve la vocation initiale qui pousse à l'action civique les humanistes du début du siècle tels Coluccio Salutati puis Leonardo Bruni, selon la conception chère à Hans Baron d'umanesimo civile.

Au fil de la correspondance et même dans l'œuvre poétique d'Eneas Sylvius Piccolomini, se dessine une image assez originale du chef idéal : soldat poète, imperator érudit. La liste des exempla tirés de l'histoire romaine ne fait pas défaut à cette longue lettre que l'on peut considérer comme un petit traité d'éducation au même titre que le De liberorum educatione,

38. Ibid., p. 602 : "Nolo te noctes diesque libros uoluere. Sed unam dumtaxat cuiuslibet diei horam exposco, quam litteris praebeas. [...] Ille autem peritus est qui naturali praeditus ingenio, uigili cura perscrutatus est magistros artium auctores, qui cum multa uiderit atque legerit librorum uolumina tum memoriae plurima commendauerit, qui non solum diuinis litteris, sed etiam secularibus sit imbutus. Ex his unum apud te uellem deligere quocum pro tuo loquereris arbitrio [...]. Ego quidem laudo aedes tuas militibus plenas esse. Hi enim sunt qui tutantur patriam et decorum est armorum gloriam principem enitere, sed nullum apud te fore uirum doctum, nec laudo nec probo. Sicut enim milites pascis ita et doctrinarum institutores nutrire posses, qui te iusti et iniusti differentias et limites edocerent uirumque redderent ex omni parte perfectum."

39. Ibid., p. 602.

40. Ibid., p. 473 : "Cum Alphonsum ego ex Bais Puteolos usque sequerer essetque illi ad me sermo de literis, ait se legisse librum Augustini de ciuitate Dei, ex latino sermone in Galliam linguam translatum, in cuius proemio scriptum esset, Regem illitteratum nihil aliud nisi asinum coronatum esse. "

4I. Voir : Collectif, L'homme de guerre au XVI siècle (Actes du colloque de l'Association Rhr, Cannes, I989), Presses universitaires de Saint-Étienne, 1992. 
avant lui le De studiis et litteris liber de Leonardo Bruni et bon nombre de traités d'éducation du Quattrocento, après lui les deux lettres de Flavio Biondo à Gianfrancesco Gonzaga. Les deux principaux exempla retenus par Piccolomini sont César et Auguste, tous deux hommes de lettres et grands généraux. Piccolomini rappelle la réception de Jules César dans l'académie des poètes ${ }^{42}$ et sa réaction bienveillante et modeste lorsqu'Accius, qu'il savait bien meilleur poète que lui, ne s'est pas levé en le voyant entrer. Pour Piccolomini, le poète et le soldat ont en commun l'amour de la gloire et de la vertu, deux qualités conciliables en la personne du chef de guerre idéal. Dans une de ses poésies de jeunesse, il exalte la figure d'un chef de guerre contemporain, Blasius Axeretus, victorieux à l'issue d'un combat naval sur Alphonse d'Aragon (lui même cité comme incarnation de cette double perfection ${ }^{43}$ ).

La vision non désintéressée de l'étude des auteurs antiques et de la rhétorique apparaît dans la lettre à Sigismond lorsqu'il analyse les compétences indispensables à un homme d'État :

Choisis pour toi un homme de grande culture et ne regarde pas à la dépense là où tu recevras un considérable avantage. Tu te demandes peut-être quel avantage? J'en énumèrerai quelques uns pour que tu ne méprises pas l'utilité des belles lettres. Une fois que tu seras devenu un homme, tu auras pour toi cet honneur et cet avantage : lorsque tu prends la parole dans un conseil, tout le monde t'écoute, dans la mesure où, à toi seul, tu en sais plus que tous les autres. Personne ne pourra te duper, personne n'osera dire que telle ou telle chose est juste ou injuste sans s'en être assuré. $\mathrm{Si}$ quelqu'un a l'impudence de te proposer quelque projet malhonnête, tu seras prompt à réfuter ses arguments. Si tu veux t'adresser au peuple, les belles lettres t'enseigneront de quelle façon il faut t'exprimer. Si tu désires ou bien faire l'éloge d'une personne ou la condamner, à la fois Quintilien et Cicéron t'y aideront. Si tu dois entreprendre une guerre et te consacrer aux armes, Végèce t'enseignera comment t'y prendre, ainsi que Tite-Live, Quinte-Curce, Justin, Lucius Florus, Suétone, Salluste et toute l'assemblée des historiens. Chez eux, tu découvriras le courage d'Alexandre le Grand, l'habileté d'Hannibal, les ruses de Fabius, la sagesse de Scipion, la discipline militaire de Jules César, l'audace de Sertorius et de Marcellus, la perspicacité de Jugurtha et les tactiques de tous ceux qui ont mené des guerres. Tu n’apprendras jamais autant par l'expérience qu'en lisant ${ }^{44}$.

42. Opera omnia, ouvr. cité, epistola CIV, p. 596.

43. Cinthia, J. Cugnoni (éd.), Atti della reale Accademia dei Lincei, anno CCLXXX, vol. 8, 1883, p. 66I (élégie XII, In Axaretum).

44. Opera omnia, ouvr. cité, p. 603 : "Sume igitur tibi grandis doctrinae aliquem uirum nec parcas expensis ubi ingens redundat emolumentum. Quaeris fortasse quod emolumentum? Edicam paucis, ne literarum utilitatem contemnas. Postquam enim uiriles attigeris annos, hoc tibi honoris et commodi erit, ut te in consilio loquente caeteri sileant, cum tu unus plus omnibus sapias : nemo te decipere poterit, nemo dicere audebit, hoc aequum et hoc iniquum, nisi uerum id esse manifeste cognouerit. Si quis praesumpserit inhonesti aliquid suadere praesto eris rationibus confutare. Si affari uolueris populum, quo pacto loquendum sit litterae te instituent. Si autem laudare aliquem aut 
Le portrait qu'il dresse de Mariano Sozzino son ancien professeur de Sienne est à cet égard très riche d'enseignements puisqu'après avoir énuméré les innombrables qualités du savant, il indique que s'il a connu à son époque de nombreux érudits, ceux-ci étaient souvent incapables de se livrer avec compétence à la gestion politique d'une cité. L'idéal du lettré qui s'engage dans la vie de la cité est défini à travers l'énumération de plusieurs grandes figures : "Ceux qui sont dignes de tous les éloges et de toute l'admiration sont les hommes qui ont servi la République sans négliger les belles lettres, comme par exemple Platon, Aristote, Démosthène, César, Cicéron, Pline, ainsi que Mécène et Auguste ${ }^{45}$. » Il n'est pas anodin que Piccolomini achève son énumération sur deux noms qui nous renvoient, plus qu’à des auteurs, à des personnalités qui ont protégé et encouragé les artistes. La recommandation est claire : "idem et tu facies si uir et princeps optimus esse uolueris. "

Dans l'humanisme actif que prône Piccolomini, la poésie constitue à la fois le pilier et le corollaire de l'action politique : elle implique, tout comme la politique, la connaissance de la rhétorique, de l'histoire et de la philosophie. La lettre CCCCII à Zbegniew Olesnicki (27 octobre I453) montre à quel point le fait que les Germains soient ignorants en matière de poésie et même hostiles à cette forme littéraire, joue en leur défaveur. La célébration de la poésie comme instrument politique qui instaure le dialogue, la paix entre les peuples est très étonnante pour le lecteur moderne :

En effet, comment les provinces pourraient-elles être pacifiées, poussées à l'amitié ou rétablies dans leurs statuts sans l'éloquence que nourrit la poésie? Ignores-tu l'importance de la correspondance, de l'histoire, de l'art oratoire? Par lettre, un roi se rapproche d'un autre roi, les cités scellent des traités, les absents dialoguent avec les absents. L'histoire nous rapporte les hauts faits, elle qui est témoin de son temps, lumière de la vérité, maîtresse de l'existence. Les orateurs tiennent le Sénat, guident les peuples, portent les lois. Mais qui peut accomplir tout cela correctement sans être imprégné par l'art poétique et par l'art oratoire ${ }^{46}$ ?

uituperare uolueris et Quintilianus et Cicero te docebit. Si bellum suscipiendum erit et armis opera danda, Vegetius modum ostendit et Liuius et Quintus Curtius et Iustinus et Lucius Florus et Suetonius et Sallustius Crispus et historicorum cuneus, in quibus et Alexandri Magni fortitudinem et Anibalis calliditatem et Fabii uersutias et Scipionis prudentiam et Iulii Cesaris disciplinam militarem et Sertorii ac Marcelli audaciam et Iugurte sagacitatem et omnium, qui res bellicas gesserunt, artes inuenies. Nunquam tam multa experiundo uidebis quam multa legendo inuenies. "

45. Ibid., p. 604 : "Illi sunt omni laude et praeconio digni qui et rei publicae seruierunt et litterarum studia non omiserunt ut et Platonem et Aristotelem et Demosthenem et Iulium et Ciceronem et Plinium et Macenatem et Augustinum fecisse comperimus. Hi namque quod ex litteris hauserant, in administranda republica exercebant. "

46. Ibid., p. 620 : "Quomodo enim pacari prouinciae et amicitiae uel conflari uel integrari possent nisi facundia foret, quam poesis alit. An nescis quantus est epistolarum usus quantus historiarum quantus orationum. Rex regi per litteras conciliatur, ciuitates foedera percutiunt, absentes cum absentibus fabulantur. Historia nobis res gestas refert quae testis est temporum, lux ueritatis est, uitae magistra. Oratores senatum regunt, populos ducunt, leges suadent. Sed quis haec bene agat nisi poeticis et oratoriis imbutus disciplinis?" 
Piccolomini rappelle ensuite les exempla issus de l'histoire grecque et romaine pour prouver que les poètes ont toujours été utiles aux grands hommes et qu'ils ont été récompensés en proportion des services rendus. Dans la virulente défense de la poésie que Piccolomini a écrite dans la lettre à Wilhelm von Stein ( $\mathrm{I}^{\text {er }}$ juin $\mathrm{I} 444$ ) en réaction aux attaques d'un juriste borné qui proclame l'inutilité de la poésie, il montre bien que la poésie, outre les charmes et les vertus que tous les hommes sensés lui reconnaissent, offre un avantage matériel certain puisque les hommes de lettres cités ont considérablement accru leur patrimoine quia poesim et oratoriam sunt secuti. Et il ajoute: "Aucun vendeur d'or ne te rapportera autant que Quintilien n'a rapporté à Leonardo d'Arezzo ${ }^{47}$. " Piccolomini connaissait la lettre de saint Jérôme (XXII, 30) qui ouvre le débat sur la compatibilité de la poésie païenne avec la foi chrétienne; un passage de la lettre à Zbegniew Olesnicki ${ }^{48}$ en est directement inspiré. La première moitié du Quattrocento est riche en défenses de la poésie, on ne peut les citer toutes, ce qui veut dire que les enjeux sont essentiels. E. Garin a montré qu'au Moyen Âge, deux conceptions de la poésie cohabitent en s'oppo$\operatorname{sant}^{49}$ : la poésie comme divertissement, fables antiques, ou bien intuition de Dieu contenue dans les Livres saints. Ces deux conceptions opposées font l'objet de débats au Trecento qui trouvent des échos dans la lettre de Piccolomini. Il cite notamment Platon traduit par Chrysoloras pour donner son interprétation du fait que Platon dans la République chasse le poète de la cité idéale. Dans la lettre au cardinal prêtre de Sainte-Prisque, le Polonais Zbigniew Olesnicki, il répond ${ }^{\text {so }}$ : «Que venez-vous m’opposer la cité de Platon qui n’a jamais existé nulle part? Pourquoi se soucier de ce que Platon chasse les poètes de sa cité qui n’a jamais existé, ni au ciel, ni sur terre? ", avant de conclure que Platon était lui-même poète.

Cet éloge de la poésie ne peut pas être envisagé séparément de l'exigence d'encyclopédisme que laissent transparaître les conseils de lecture que les humanistes prodiguent aux jeunes princes ou aux femmes promises à un grand avenir. Bruni ${ }^{\text {st }}$, déjà, dans les Dialoghi dès le début du siècle, avait

47. Ibid., p. 619, epistola CXI : "Nec tibi unquam tantum auri speculator praebebit quantum Quintilianus Aretino tribuit. "

48. Ibid., p. 939 (Lettre de novembre 1463).

49. E. Garin, Moyen Âge et Renaissance, Paris, Gallimard, 1989, p. 4I-55.

50. Opera omnia, epistola CCCCII, p. 938 : "Quid uos mihi ciuitatem Platonis obiicitis? quae nulla umquam in prouincia sedem reperit, quid curet ex ea se ciuitate Poetarum choros exulare, quae neque in coelo, neque in terra uisa est umquam? At cum poetarum fictiones bicipitis plerumque Parnasi somnia referant, non possum non admirari cur somniatores e ciuitate somniata Plato deduxerit? Cur ego non exulare ea ciuitate uoluerim, quam conditor eius inhabitare non possit? Aut equidem suam ciuitatem necesse fuisset Platonem relinquere, aut secum introducere poetas, cum fuerit ipse poeta."

5I. Dialogus II, \$70. 
énoncé des exigences auxquelles font écho les qualités requises pour faire un bon secrétaire pontifical selon Piccolomini :

Je crois qu'un poète digne de ce nom doit avoir trois qualités : la capacité d'invention la finesse de l'expression, une culture étendue. La première de ces qualités est propre aux poètes, ils partagent la deuxième avec les orateurs et la troisième avec les philosophes et les historiens.

Pour Piccolomini, la littérature est dans la vie et l'action consiste précisément à mettre concrètement en application les enseignements que l'on a retirés de la lecture. Les deux entités sont indissociables dans l'idéal de l'honnête homme au service de sa ville, de sa principauté ou de son pays : il ne saurait être question de louer un homme de lettres enfermé dans son cabinet de travail, mais il n'est pas question non plus de faire l'éloge du prince actif et combattant qui ne prendrait ni le temps de l'étude, ni le temps de la réflexion. Chez Piccolomini comme chez de nombreux humanistes de cette période charnière que constitue le Quattrocento, on peut relever des intuitions géniales et des progrès spectaculaires en même temps que des lourdeurs et des archaïsmes qui le relient au passé. On lit que Piccolomini a mis son génie au service de causes rétrogrades, qu’il est encore, par certains aspects, un homme du Moyen Âge. Comment ne le serait-il pas? Il serait plus intéressant, de se demander si l'idéal du lettré en action, servant les belles lettres et la cité non seulement sans hiatus mais de façon complémentaire, ne correspond pas au portrait exact de l'humaniste même qui brosse ce portrait pour un prince italien ou un empereur germanique. N'est-il pas frappant de constater que la biographie de Piccolomini souligne depuis l'époque de ses études jusqu'à son engagement politique auprès des grands, par la suite, comme cardinal puis comme pape, une tentative de conciliation constante entre les impératifs de la foi, les apports des studia humanitatis et le dévouement envers sa patrie menacée par les ennemis de la chrétienté et de l'héritage gréco-latin? Laissons le dernier mot à Piccolomini devenu Pie II lorsqu'il apprit la chute de Constantinople : "C'est une deuxième mort pour Homère, un second trépas pour Platon : où pourrons-nous désormais rechercher les œuvres géniales des philosophes et des poètes grecs? La source de la poésie s'est tarie... ( (Lettre à Nicolas V le I2 juillet I453.) 
